\title{
Der Stellenwert der Schwerbehindertenvertretung in der betrieblichen Inklusion
}

Die Veränderungen aufgrund des demografischen Wandels und der Anhebung des Rentenalters haben Auswirkungen auf die Arbeits- und Beschäftigungsfähigkeit. Mit höherem Alter und vermehrten Belastungen in der Arbeitswelt steigt das Risiko von Leistungseinschränkungen und Langzeiterkrankungen. Eine zentrale Chance zur Fachkräftesicherung ist die Schaffung eines modernen Inklusionsverständnisses, das darauf abzielt, dass von Behinderung bedrohte und schwerbehinderte Menschen Chancengleichheit erleben und einen gleichberechtigten Zugang zur Arbeitswelt haben. Diese Zielvision eines inklusiven Arbeitsmarktes verdeutlicht den Stellenwert einer betrieblichen Interessenvertretung schwerbehinderter Beschäftigter.

\section{Einleitung}

Das idealtypische Bild einer inklusiven Arbeitswelt, in der Menschen mit Behinderung selbstverständliche Mitglieder einer Belegschaft sind, und als „a work environment that is open, inclusive and accessible to persons with disabilities" bezieht sich auf die Forderung der Konvention der Vereinten Nationen über die Rechte von Menschen mit Behinderung (UN-BRK) in Artikel 27 (1) (BMAS 2011). Diese gesetzliche Verpflichtung zielt auf gleichberechtigte und vollwertige Partizipation und Chancengleichheit, indem sich insbesondere die Rahmenbedingungen der Arbeitswelt verbessern sollen und nicht vordringlich das Individuum, also der behinderte Arbeitnehmer oder die Arbeitnehmerin, sich anpassen soll. Damit wird ein Paradigmenwechsel von Integration zu Inklusion gefordert.

Im betrieblichen Alltag bedeutet die Forderung nach einer inklusiven Arbeitswelt die Sicherstellung von Chancengleichheit, die Gewährleistung eines gleichberechtigten Zugangs zu allen Bereichen betrieblichen Geschehens sowie ein Verbot von negativer Diskriminierung (ILO 2007). Die frühzeitige und umfassende Identifizierung von Bedarfen und die Einleitung geeigneter präventiver und rehabilitativer Maßnahmen im betrieblichen Setting sind Aufgaben, die eine systematische Kommunikation und einen Erkenntnis- und Wissenstransfer zwischen den Fachkräften der Rehabilitation und Prävention, den Sozialversicherungsträgern sowie innerbetrieblichen Akteuren und Akteurinnen wie dem Betriebsarzt bzw. der -ärztin, den Inklusionsbeauftragten, dem Betriebs- bzw. Personalrat (BR/PR) und der Schwerbehindertenvertretung (SBV) erfordern. Der SBV kommt in diesem Rahmen eine besondere Bedeutung zu, da sie als betriebliche Interessenvertretung von Menschen mit Behinderung ein „Motor der Inklusion“ (DGB 2015) sein und in ihrer Rolle als Vertrauensperson der schwerbehinderten Menschen inklusionswirksam agieren kann. Im Folgenden wird zwischen Schwerbehindertenvertretung als dem institutionalisierten Amt und der Person, die dieses Amt innehat, unterschieden.

Eine systematische Darstellung der Arbeit der SBVen bzw. Vertrauenspersonen in der betrieblichen Praxis ist bisher aufgrund eines fehlenden Wissenschafts-Praxis-Diskurses nicht möglich. Für ein besseres Verständnis des Stellenwerts der SBVen wird daher in diesem Aufsatz auf Grundlage des Projekts „Schwerbehindertenvertretungen: Allianzpartner in Netzwerken“" (gefördert durch die Hans-Böckler-Stiftung) die Situation der SBVen in Deutschland in ihrer alltäglichen Arbeit dargestellt. $\mathrm{Zu}$ Beginn wird die Rolle der Vertrauensperson rechtlich eingeordnet und im Zusammenhang mit möglichen Spannungsverhältnissen theoretisch betrachtet (Abschnitt 2). 
Vor diesem Hintergrund folgt die Auseinandersetzung mit empirischen Ergebnissen zu Merkmalen der SBVen in Deutschland, zu ihren Tätigkeiten und Kooperationsbeziehungen sowie zu möglicherweise auftretenden Rollenkonflikten (3). Abschließend werden Perspektiven für die Auflösung möglicher Konflikte und für eine Stärkung der Rolle der Vertrauenspersonen diskutiert (4). Der Aufsatz leistet damit einen Beitrag zur Aufwertung der Arbeit der SBVen in der betrieblichen Inklusion von gesundheitlich beeinträchtigten Personen. Dazu wird auch auf Ergebnisse einer empirischen Untersuchung von SBVen zurückgegriffen, die in Deutschland erstmals durchgeführt wurde.

\section{Rechtlicher und theoretischer Hintergrund}

\subsection{Betriebliche Interessenvertretung schwerbehinderter Menschen im deutschen Recht}

Die Selbstvertretung der schwerbehinderten Beschäftigten ist ein wichtiger Schritt für die Schaffung einer inklusiven Arbeitswelt und wird in der UN-BRK mit dem aus der internationalen Behindertenbewegung stammenden Leitsatz "Nothing about us without us" zum Ausdruck gebracht (Degener 2009; Düwell 2018). Im internationalen Vergleich ist eine betriebliche Interessenvertretung von Menschen mit Behinderung nur im deutschsprachigen Raum rechtlich gesichert. Ein der deutschen SBV vergleichbares rechtlich verankertes Amt findet sich im europäischen Raum ausschließlich in Österreich mit der Behindertenvertrauensperson (BVP) (Bernhard et al. 2011).

Die SBV ist im deutschen Recht als eine Interessenvertretung für Menschen mit Behinderungen in der Arbeitswelt installiert: „In Betrieben und Dienststellen, in denen wenigstens fünf schwerbehinderte Menschen nicht nur vorübergehend beschäftigt sind, werden eine Vertrauensperson und wenigstens ein stellvertretendes Mitglied gewählt, das die Vertrauensperson im Falle der Verhinderung vertritt“, heißt es in $\$ 177$ des Sozialgesetzbuchs (SGB) IX. In immer mehr Betrieben und Dienststellen wählen schwerbehinderte und ihnen gleichgestellte Personen ihre Interessenvertretung (Schuster 2014). Auch politisch erfährt die SBV einen Bedeutungszuwachs - nicht zuletzt wurde der SBV durch die neu normierte Inklusionsvereinbarung „ein betriebliches Regelungsinstrument an die Hand gegeben“ (Kohte/Liebsch 2019, S.4), das die Inklusionsarbeit verbindlich steuern und die Beschäftigungssituation schwerbehinderter und gleichgestellter Menschen effektiv verbessern kann.

In Deutschland sind die Aufgabenbereiche, Rechte und Pflichten der SBV im SGB IX bestimmt: Der Aufgabenbereich umfasst vorrangig die Förderung der Teilhabe schwerbehinderter oder ihnen gleichgestellter Menschen im Betrieb sowie die Vertretung der Interessen dieser Gruppe (\$178, Abs.1 SGB IX). Die SBV übt eine Beratungstätigkeit für Anliegen dieser Gruppe aus und fördert im Besonderen durch persönliche Beratung und Belegschaftsnähe ein Vertrauensverhältnis zu den Kollegen und Kolleginnen. Die SBV „hat nicht nur die besonderen Interessen der schwerbehinderten Menschen zu vertreten, sondern soll diesen nach $\$ 178$ Abs.1 Satz 1 SGB IX beratend und helfend zur Seite stehen. Im Rahmen dieser auf Einzelfallhilfe ausgelegten Unterstützungsaufgabe hat sich die SBV auch um die besonders schutzbedürftigen Beschäftigten zu kümmern“ (Düwell 2018, S.110). Sie leistet somit neben der Vertretung der Interessen ihrer Zielgruppe eine weitgehende Beistandsaufgabe. Im Zuge der Beratung leitet die SBV auch Maßnahmen zur Förderung der beruflichen Teilhabe ein und steht in engem Austausch mit zuständigen Stellen wie der Agentur für Arbeit oder dem Integrationsfachdienst. Ebenso wirkt sie an der Einrichtung und Durchführung des Betrieblichen Eingliederungsmanagements für die schwerbehinderten und gleichgestellten Beschäftigten mit ( $\$ 167$, Abs. 2 SGB IX) und klärt gemeinsam mit der betroffenen Person und der Unternehmensleitung, wie eine Arbeitsunfähigkeit überwunden, erneuter Arbeitsunfähigkeit vorgebeugt und der Arbeitsplatz erhalten werden kann. Die SBV unterstützt außerdem die Geschäftsführung bei individuellen und generellen Fragestellungen zur beruflichen Teilhabe schwerbehinderter und ihnen gleichgestellter Menschen und überprüft die Einhaltung gesetzlicher Pflichten. Darüber hinaus ist sie auch Ansprechpartnerin für den BR/PR sowie für die bzw. den Inklusionsbeauftragte ${ }^{\star} n$ der Unternehmensleitung und agiert mit diesen gemeinsam im Rahmen eines betrieblichen Integrationsteams ( $\$ \$ 176,178$ und 181 SGB IX). Das Team wirkt bei der Erarbeitung und beim Abschluss einer Inklusionsvereinbarung, bei Fragestellungen zur Prävention und innerhalb des Betrieblichen Eingliederungsmanagements mit (BIH 2018b).

Zur Wahrnehmung der beschriebenen Aufgaben finden sich im Gesetz Anhörungs-, Initiativ- und Beteiligungsrechte ( $\$ 178$ SGB IX). Zu ersteren gehört die Pflicht der Arbeitgeber*innen, die SBV in allen Angelegenheiten, die einen einzelnen oder die schwerbehinderten Menschen als Gruppe berühren, unverzüglich und umfassend $\mathrm{zu}$ unterrichten und vor einer Entscheidung anzuhören. Eine getroffene Entscheidung muss der SBV unverzüglich mitgeteilt werden. Beispielsweise ist die Kündigung eines schwerbehinderten Menschen, die das Unternehmen ohne SBV-Beteiligung ausspricht, unwirksam. Durch das Initiativrecht hat die SBV die Möglichkeit, Verhandlungen über eine Inklusionsvereinbarung einzuleiten. Auf Antrag der SBV und unter Beteiligung des BR/PR ist die Unternehmensleitung verpflichtet, solche Verhandlungen aufzunehmen ( $\$ 166$ SGB IX). Beteiligungsrechte gewähren der SBV die Möglichkeit zur Teilnahme an Sitzungen des BR/PR oder anderer Gremien wie eines Personalrekrutierungsteams. Bei Themen, die schwerbehinderte Bewer- 
ber*innen oder Mitarbeiter ${ }^{\star}$ innen betreffen, muss die SBV informiert und zur Teilnahme eingeladen werden, damit sie ihre Aufgaben wahrnehmen kann.

Für die Ausübung des betrieblichen Ehrenamts der SBV sind unterschiedliche Freistellungsmöglichkeiten vorgesehen, abhängig von der Anzahl der schwerbehinderten Menschen im Betrieb oder der Dienststelle. Sind in der Regel wenigstens 100 schwerbehinderte Menschen beschäftigt, wird die SBV auf ihren Wunsch für ihre Aufgaben vollständig freigestellt ( $\$ 179$ Abs. 4 SGB IX). Sind die Voraussetzungen für vollständige Freistellung nicht erfüllt (oder wird sie nicht gewünscht), ist Teilfreistellung möglich, sodass die Tätigkeit als SBV neben der operativen Arbeitstätigkeit ausgeübt wird. Insbesondere für solche Vertrauenspersonen gilt, dass „,von einer hohen persönlichen Anstrengung bzw. einem hohen persönlichen Engagement ausgegangen werden kann" (Bernhard 2008, S. 7of.). Das Amt der SBV und Ämter im BR bzw. PR sind miteinander vereinbar; weder Betriebsverfassungsgesetz (BetrVG) noch SGB IX schließen eine Personalunion aus (BIH 2018b). Ansonsten sind die Betriebs- und Personalräte - wie die Arbeitgeberseite auch - durch $\$ 182$ SGB IX zu enger Zusammenarbeit mit der SBV aufgerufen.

\subsection{Rollen-Set der Schwerbehindertenvertretung}

Die SBV kann mit verschiedenen Akteur*innen den Weg hin zu einer inklusiven Arbeitswelt in einem partizipativen Prozess, in dem Synergien geschaffen und genutzt werden, gemeinsam gehen (INQUA 2011). Mit der Zusammenarbeit entstehen durch die verschiedenen Interessen der einzelnen Personen(gruppen) aber auch Spannungsverhältnisse. Die Handlungsweisen der SBV können sich themenbedingt und kontextspezifisch zwischen Konflikt und Kooperation bewegen. Schumann et al. (2008, S. 255; zitiert nach Stracke/Nerdinger 2009) verweisen darauf, dass Kooperation und Konflikt nebeneinander bestehen und nicht als „gegensätzliche, sich ausschließende Strategien gedacht" sind. Je nach Situation kann dabei aber die eine oder andere Seite überwiegen.

Aufgrund der Institutionalisierung des Amtes der SBV liegt es auf der Hand, dass sich die Vertrauenspersonen einer Vielzahl von Erwartungen ausgesetzt sehen. In ihrem Amt als SBV sind sie vorrangig am Schnittpunkt zweier Interessengruppen tätig - den schwerbehinderten und ihnen gleichgestellten Beschäftigten und der Geschäftsführung. Die Beziehungen der Vertrauensperson zu den Kolleginnen sowie zur Geschäftsführung sind jeweils durch eigene Themen, Anforderungen und Problemfelder charakterisiert. Darüber hinaus muss die SBV in ihrer Arbeit, im Sinne der betrieblichen Prävention, auch die Bedarfe der nicht direkt von gesundheitlichen Einschränkungen betroffenen Belegschaftsmitglieder berücksichtigen. Ergänzend agieren viele Vertrauenspersonen, wenn sie nur teilweise freigestellt sind, in einem Spannungsverhältnis aufgrund der Tatsache, dass sie neben dem betrieblichen
Ehrenamt ihre operative Tätigkeit und, falls sie ebenfalls Mitglied des BRes/PRes sind, zudem Funktionen aus diesem Amt ausüben müssen.

In Anlehnung an Merton (1973) bezeichnen wir die unterschiedlichen Verhaltenserwartungen, denen sich die Vertrauenspersonen gegenübersehen, als Rollen und die Menge der mit einer Position verbundenen Rollen als Rollen-Set. Um das hier relevante Rollen-Set zu bestimmen, sind zum einen die Bezugspersonen und -gruppen sowie deren Interessen und Erwartungen ins Auge zu fassen (Abbildung 1). Hieraus wird ersichtlich, dass die jeweiligen Personen(gruppen) durch ihre eigenen Interessen und Erwartungen geprägt sind und diese in Form von Rollenerwartungen an den Rollenträger (in diesem Fall die Vertrauensperson) herantragen. Zum anderen sind auch Erwartungen zu berücksichtigen, die nicht durch die verschiedenen Bezugspersonen und -gruppen, sondern durch das Einnehmen verschiedener Rollen in einer Person entstehen. Unter Umständen sind mehrere Rollen zur gleichen Zeit in einer Person vereinigt (Abbildung 2). Beide Aspekte zusammen machen die Rolle der SBV aus.

\section{ABBILDUNG 1}

\section{Rollen-Set der Schwerbehindertenvertretung}

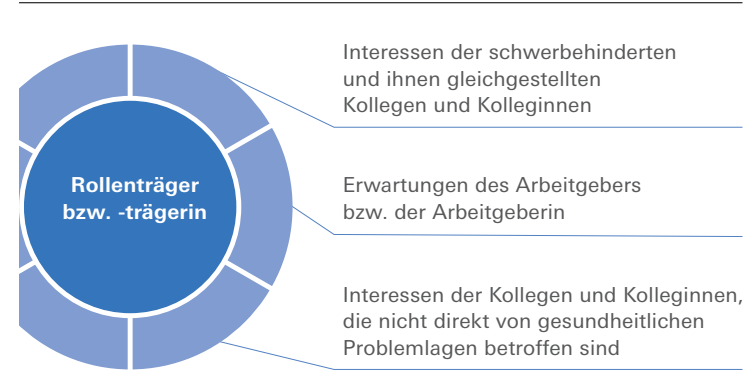

Quelle: Eigene Darstellung

ABBILDUNG 2

\section{Rollen-Set der Vertrauensperson}

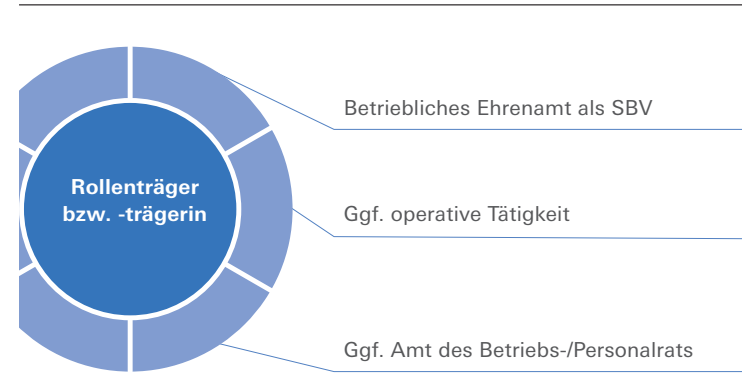

Quelle: Eigene Darstellung 
An einen Rollenempfänger bzw. eine Rollenempfängerin gerichtete Erwartungen sind für gewöhnlich nicht eindeutig. Zudem unterscheiden sich häufig die Erwartungen, die aus verschiedenen Positionen gesendet werden (Nerdinger 2012). Nach dem Modell des Rollenstresses (Kahn 1978; Kahn/Byosiere 1992) können durch inkonsistente Informationen und unvereinbare Rollenanforderungen Rollenkonflikte entstehen. Von Intra-Rollenkonflikten spricht man, wenn an Rollenträgerinnen Forderungen und Erwartungen gestellt werden und diese miteinander kollidieren oder einen uneindeutigen bzw. widersprüchlichen Charakter haben. Durch das Ausfüllen verschiedener Rollen in einer Person können außerdem Inter-Rollenkonflikte auftreten, wenn die Anforderungen der verschiedenen Rollen unterschiedlich sind und der bzw. die Rollenträger ${ }^{\star}$ in sie zu vereinbaren versucht (Kauffeld 2004).

Durch die Vertretung der Zielgruppe schwerbehinderter und mit gesundheitlichen Problemlagen konfrontierter Beschäftigter sowie der vertrauensvollen Zusammenarbeit mit ihnen ist die SBV in einer ständigen Vermittlungs- und Brückenposition und kann so einen entscheidenden Vernetzungsknoten zwischen den betreffenden Beschäftigten, der Geschäftsführung und weiteren inner- und außerbetrieblichen Akteur*innen bilden (BIH 2018a). Daher ist es wünschenswert, die Möglichkeiten auszuschöpfen, mittels derer die SBVen diese Funktion noch besser erfüllen können, und ihre Rolle zu stärken (Kohte/Liebsch 2019); dazu kann auch vertieftes empirisches Wissen über die Strukturen, Probleme und Hemmnisse der gegenwärtigen Praxis nützlich sein.

\section{Empirische Untersuchung zur Situation der Schwerbehinderten- vertretung in Deutschland}

\subsection{Forschungsdesign}

Mit dem Ziel, die Situation der SBV in ihrem betrieblichen Alltag zu untersuchen, wurden Daten in vier Schritten erhoben. Im ersten Schritt wurden in der zweiten Jahreshälfte 2017 deutschlandweit SBVen aller Branchen und Unternehmensgrößen schriftlich mit einem Online-Survey befragt, anschließend wurden für eine tiefergehende Analyse leitfadengestützte Interviews mit einzelnen Vertrauenspersonen sowie drittens Fokusgruppendiskussionen mit Vertrauenspersonen und ihren inner- und außerbetrieblichen Kooperationspartner*innen realisiert. Im Anschluss wurde im vierten Schritt eine Zukunftswerkstatt mit Akteuren und Akteurinnen aus der betrieblichen Praxis durchgeführt.

Die deutschlandweite Befragung diente der Erhebung von Merkmalen der Situation der SBV sowie der inhaltli- chen Differenzierung von Tätigkeitsfeldern und Kooperationsbeziehungen. Die Operationalisierung fand mithilfe von Expert*inneninterviews mit Vertrauenspersonen sowie mithilfe von Auswertungen relevanter Literatur und der gesetzlichen Grundlagen statt. Die Kontaktaufnahme zur Zielgruppe erfolgte über Gewerkschaften, Inklusionsbzw. Integrationsämter sowie über verschiedene Arbeitsgemeinschaften der SBVen, welche mithilfe von E-Mails, Newslettern, Zeitschriften und sozialen Netzwerken zur Teilnahme aufriefen. Eine möglichst breite Beteiligung wurde nicht zuletzt deshalb angestrebt, weil es die Statistiken der Integrations- bzw. Inklusionsämter nicht zulassen, Aussagen über die Grundgesamtheit der SBVen in Deutschland zu treffen.

Um die Zusammenarbeit der Vertrauenspersonen mit ihren Kooperationspartner*innen mehrperspektivisch beleuchten und Kooperations- und Spannungsfelder identifizieren zu können, fanden leitfadengestützte Interviews mit vier Vertrauenspersonen deutscher Betriebe und vier Fokusgruppendiskussionen mit diesen und ihren innerund außerbetrieblichen Kooperationspartner ${ }^{\star}$ innen statt. Das Sampling zu den Interviews erfolgte deduktiv auf Grundlage festgelegter Kriterien (top-down) mit der Zielstellung, eine möglichst heterogene, in bestimmten Merkmalen kontrastierte und somit informative Stichprobe zu generieren (Flick et al. 2017). ${ }^{1}$

Die vier Fokusgruppendiskussionen erfolgten mit je einer Vertrauensperson und ihren Kooperationspartner*innen. An zwei Fokusgruppendiskussionen (mit drei und vier Teilnehmenden) nahmen ausschließlich innerbetriebliche Akteur*innen, an zwei weiteren (mit vier und sieben Teilnehmenden) ausschließlich außerbetriebliche Akteur ${ }^{\star}$ innen teil. Für die Auswahl wurde auf die vorherigen leitfadengestützten Interviews zurückgegriffen, um sicherzustellen, dass die Beteiligten ausreichenden Kontakt miteinander haben und dementsprechend Aussagen über die Zusammenarbeit treffen konnten.

Die gewonnenen Erkenntnisse der drei ersten Schritte wurden abschließend mit betrieblichen Akteur*innen in Form einer Zukunftswerkstatt (mit 14 Teilnehmenden) diskutiert. Das Ziel dieses Schritts lag darin, Chancen und Risiken der Zusammenarbeit von SBV und BR/PR in der betrieblichen Praxis zu identifizieren.

1 Einbezogen wurden demgemäß Vertrauenspersonen mit und ohne gleichzeitiger Ausübung des BR/PR-Amtes und aus Betrieben unterschiedlicher Größe - damit auch mit unterschiedlichen Anzahlen zu betreuender schwerbehinderter bzw. gleichgestellter Beschäftigter und, analog dazu, mit voller oder Teil-Freistellung. Um diese Varianz dennoch vor vergleichbaren Rahmenbedingungen analysieren zu können, wurde die Stichprobe auf Personen aus ähnlichen Branchen (Bergbau, Chemie und Energie) eingeschränkt. 


\subsection{Ergebnisse}

3.2.1 Verbreitung und Zusammensetzung der Schwerbehindertenvertretungen

An der schriftlichen Befragung haben sich 1552 Personen beteiligt. Die erhobenen Merkmale der Vertrauenspersonen - Alter, Geschlecht, Schwerbehindertenstatus, Betriebsgröße, Freistellung, gleichzeitige Ausübung des BR/ PR-Amtes, Gewerkschaftsmitgliedschaft - sind bisher nicht systematisch erforscht worden. Es liegen somit keine Vergleichsdaten und keine Angaben zur Grundgesamtheit vor.

Mehr als die Hälfte der Befragten (60\%) sind Männer, rund $70 \%$ sind 51 Jahre oder älter. $73 \%$ geben an, selbst einen Schwerbehindertenstatus zu haben. Rund $88 \%$ der SBV arbeiten in größeren oder Großunternehmen mit mehr als 249 Beschäftigten, $12 \%$ in kleineren und mittleren Unternehmen mit 50 bis 249 Beschäftigten. Gut drei Viertel (77\%) der Vertrauenspersonen gehen neben ihrem betrieblichen Ehrenamt auch einer operativen Tätigkeit nach und sind für die SBV-Arbeit nur teilweise freigestellt. Rund $44 \%$ der Befragten üben sowohl das Amt der SBV als auch ein BR/PR-Amt aus. Knapp die Hälfte (47\%) ist Mitglied einer Gewerkschaft.

\subsubsection{Tätigkeitsbereiche und Kooperationsbeziehungen der Schwerbehindertenvertretung}

Die Befragten wurden gebeten, die Anteile ihrer Arbeitszeit im Amt der SBV anzugeben, die sie für unterschiedliche Tätigkeitsbereiche durchschnittlich aufwenden. Dabei zeigt sich, dass die Vertrauenspersonen

- etwa $12 \%$ der Zeit mit ihrer Etablierung im Ehrenamt verbringen (sich bei den Beschäftigten bekannt machen, sich Wissen zum Amt aneignen, Arbeitsmittel organisieren etc.),

- rund $22 \%$ der Zeit für Interaktionen mit der Unternehmensleitung verwenden (z.B. bei der Überwachung der Einhaltung gesetzlicher Verpflichtungen),

- ebenfalls rund $22 \%$ der Zeit für Interaktionen mit anderen Akteurinnen benötigen (z.B. bei der Mitwirkung an Sitzungen und Ausschüssen sowie im Austausch mit möglichen Kooperationspartner*innen) sowie

- den Löwenanteil von etwa $43 \%$ der Zeit der direkten Arbeit mit schwerbehinderten und ihnen gleichgestellten sowie solchen Menschen widmen, die einen Antrag auf Anerkennung ihrer Behinderung gestellt haben (hier führen sie z. B Beratungsgespräche durch und unterstützen Menschen in gesundheitlichen Problemlagen bei der Antragsstellung).

Die Zeitanteile weisen bei den Betriebsgrößenklassen keine signifikanten Unterschiede auf.

Bezüglich der Zusammenarbeit mit Kooperationspartner*innen ergibt die Befragung, dass die SBV durchschnittlich mit rund sechs inner- und vier außerbetrieb- lichen Akteur*innen kooperiert. Die dabei aufgewendeten Zeitanteile geben Hinweise auf deren Stellenwert: Innerbetrieblich arbeiten sie am meisten mit dem BR/ PR zusammen, gefolgt von der bzw. dem Inklusionsbeauftragten der Unternehmensleitung, der bzw. dem Vorgesetzten, dem Betriebsarzt bzw. der Betriebsärztin, dem betrieblichen Integrationsteam/BGM-Team, der Fachkraft für Arbeitssicherheit und dem bzw. der Disability Manager ${ }^{\star}$ in. Außerbetrieblich arbeiten sie vorrangig mit der Versorgungsverwaltung, dem Integrations- bzw. Inklusionsamt inklusive technischem Beratungsdienst sowie dem Integrationsfachdienst zusammen, gefolgt von der Rentenversicherung, der Agentur für Arbeit, Anwält ${ }^{*}$ in nen und Gerichten sowie der Unfallversicherung und der Krankenkasse.

\subsubsection{Rollenkonflikte in der SBV-Arbeit}

Die Zusammenarbeit mit verschiedenen Akteur*innen kann zwar eine Unterstützungsmöglichkeit darstellen, aber auch eine Herausforderung vor allem dann sein, wenn die SBV mit widersprüchlichen Interessen und Erwartungen von verschiedenen Seiten konfrontiert ist. Die Befragung zeigt dazu, dass sich $21 \%$ der Vertrauenspersonen in sehr hohem bzw. in hohem Maß und $53 \%$ zum Teil widersprüchlichen Anforderungen ausgesetzt fühlen. In den als widersprüchlich erlebten Anforderungen drücken sich die Ambivalenzen der erwähnten Vermittlungs- und Brückenposition aus, wie sich in den leitfadengestützten Interviews und den Fokusgruppendiskussionen zeigte.

Für die große Mehrheit von Vertrauenspersonen, die nicht vollständig von ihrer operativen Tätigkeit freigestellt sind, ergibt sich ein Inter-Rollenkonflikt, wie sich z. B. in folgender Passage aus einem Interview zeigt:

"Ich habe 5 Stunden in der Woche für die Arbeit als Vertrauensperson. Da kommen schon viele Menschen mit Problemen zu mir. [...] Da ist es für mich schwer, alles unter einen Hut zu bekommen. [...] Die Kollegen stöhnen dann schon und müssen an manchen Stellen meine Arbeit mitmachen."

Intra-Rollenkonflikte spüren auch jene Vertrauenspersonen, die zugleich ein BR/PR-Mandat ausüben, wie zwei andere Interviewpassagen illustrieren:

"Bei uns wird das kritisch betrachtet, weil die Anliegen doch oftmals nichts ganz deckungsgleich sind und wir haben das oft so, dass tatsächlich der Betriebsrat dann so emotional schon mal denkt, dass Menschen mit Schwerbehinderung bevorzugt würden gegenüber den normalen Beschäftigten [...].“

„Es war zum Beispiel bei Einstellungsgesprächen die Problematik, dass der Betriebsrat für Bewerbungen von Mitarbeitern, die schon im Hause waren, sich eingesetzt hat. Die Schwerbehindertenvertretung sich aber grundsätzlich natürlich für die, die sich beworben haben mit Behinderung eingesetzt hat, um die einzustellen bei gleicher Eignung. Und das gab schon einen Konflikt ohne Ende und da musste man sich entscheiden." 
Solche Konflikte lassen sich durch einen offensiven Umgang mit der eigenen Rolle abmildern, wie eine Vertrauensperson in der Zukunftswerkstatt empfiehlt:

„Also wenn ich in ein Gespräch reingehe, dann beschreibe ich meinem Gegenüber erst mal meine Rolle, weil ich vertrete ja verschiedene Rollen. Und wenn ich zum Beispiel in einem Konfliktgespräch vorher sage, was meine Rolle ist, was meine Aufgabe ist, dann gehen die immer emotionslos über die Bühne."

\subsection{Handlungspraktische Konsequenzen}

Die Ergebnisse ermöglichen neue Einblicke, weil die Kenntnisse über die Situation der SBV aufgrund fehlenden systematisch gesammelten Datenmaterials bisher sehr lückenhaft waren. In der sozio-demografischen Dimension fällt vor allem auf, dass der Großteil der befragten Vertrauenspersonen über 50 Jahre alt ist. Diese Altersstruktur ist plausibel vor dem Hintergrund, dass die Bereitschaft, ein betriebliches Ehrenamt $\mathrm{zu}$ übernehmen, vermehrt erst entsteht, wenn die Beschäftigten schon länger im Betrieb tätig sind, die betrieblichen Strukturen kennen und darüber hinaus - ggf. bedingt durch die eigene Lebensgeschichte - für gesundheitliche Themen im Arbeitskontext sensibilisiert sind. Ungeachtet dessen drängt sich die Frage nach der Rekrutierung von Nachwuchs für das betriebliche Ehrenamt auf. Erforderlich ist Aufklärungsarbeit im Hinblick auf das Amt der SBV, um es den Arbeitnehmer*innen besser bekannt zu machen.

Die Ergebnisse über die Zeitkontingente verdeutlichen überdies, dass die Arbeit der SBV durch eine große Belegschaftsnähe gekennzeichnet ist. Es bestätigt sich, dass die SBV einer weitgehenden Unterstützungsaufgabe nachgeht. Unter der Zielstellung der Schaffung einer inklusiven Arbeitswelt sollte die Eigenständigkeit der Rolle der SBV weiter gefördert werden, um dem Grundsatz der UN-BRK "Nothing about us without us" stärker Geltung zu verschaffen. Auch ist Düwell (2016, S.65) darin zuzustimmen, dass diese „zusätzliche Helfer- und Unterstützungsfunktion [...] ein höheres Maß an Informationen voraussetzt, als es die reine Interessenvertretung derjenigen erfordert, die keiner Hilfe und Unterstützung bedürfen“. Die SBV sollte im Hinblick auf Sozial- und Beratungskompetenzen geschult werden, um so die Unterstützung der besonders schutzbedürftigen Beschäftigten überhaupt leisten zu können.

Aufgrund möglicher Synergieeffekte eröffnet die $\mathrm{Zu}$ sammenarbeit mit verschiedenen Akteure ${ }^{\star}$ innen für die SBV die Chance, als „Motor der Inklusion“ zu wirken, aber stets besteht das Risiko von Inter- und Intra-Rollenkonflikten. Bei nicht vollständig freigestellten Vertrauenspersonen wird dieses Risiko durch das gleichzeitige Ausüben der operativen Tätigkeit und des betrieblichen Ehrenamtes erhöht. Die Lösung der Rollenkonflikte verlangt von der SBV, die an sie herangetragenen Erwartungen abzuwägen und widersprüchliche Forderungen so $\mathrm{zu}$ beeinflussen, dass sie die eigene Arbeit erfolgreich gestalten können. Um ein professionelles Selbstverständnis in Bezug auf die eigene Rolle zu etablieren, können sowohl individuelle Beratungs- als auch Gruppenangebote gemeinsam mit anderen SBV en helfen, wo die gestellten Erwartungen transparent gemacht und Möglichkeiten der Rollengestaltung und -stärkung entwickelt werden (Schiersmann/Thiel 2018). Darüber hinaus können Handlungsleitfäden, die Gelingensfaktoren und Konfliktfelder in der Zusammenarbeit vor dem spezifischen Hintergrund der jeweiligen Akteurinnen diskutieren, zur Rollenklärung sowie einer erfolgreichen Zusammenarbeit beitragen (Niehaus et al. 2019). Bei der Entwicklung von Maßnahmen sollte weiter berücksichtigt werden, dass die Rollenkonflikte häufig Personen betreffen, die selbst von gesundheitlichen Problemlagen betroffen sind (wie erwähnt, geben knapp drei Viertel der befragten SBVen an, eine Schwerbehinderung zu haben); die Maßnahmen sollten also auch Aspekte des Umgangs mit den eigenen gesundheitlichen Einschränkungen umfassen.

Im Hinblick auf Spannungsverhältnisse, die sich bei einer Personalunion von SBV- und BR/PR-Amt ergeben können, bedarf es einer besonderen Klärung. Das zusätzliche Ausüben des Amtes des BRes/PRes kann für die SBV vor dem Hintergrund der stärkeren Rechte des BRes/PRes von Vorteil sein, um den eigenen Stellenwert im Betrieb ausbauen zu können. Zugleich haben wir darauf hingewiesen, dass die Arbeit in dieser Doppelfunktion auch zu Rollenkonflikten führt. Hier stellt sich die Frage, inwiefern sowohl die SBV selbst als auch die BR/PR-Kolleg*innen diesen Konflikten präventiv entgegenwirken können. Gemeinsame Weiterbildungen für SBV- und BR/ PR-Kolleg*innen können zu Transparenz und Akzep$\tan z$ der eigenen und jeweils anderen Rollen führen und so das eigene Handeln im Hinblick auf die Abgrenzung der beiden Ämter voneinander optimieren. Unabhängig davon, ob die Ämter in Personalunion ausgeübt werden oder nicht, sollte die kooperative Zusammenarbeit von SBV und BR/PR gefördert werden, um die Stärken der jeweiligen Ämter zu bündeln. Nicht zuletzt weil auch der BR/PR die Eingliederung schwerbehinderter Menschen fördern soll ( $\$ 80$ BetrVG) und die beiden Interessenvertretungen somit ähnliche Ziele verfolgen, ist es im Sinne der Beschäftigten wichtig, dass SBV und BR/PR eine abgestimmte Politik verfolgen (Splanemann 2002).

\section{Fazit}

Vor dem Hintergrund der diskutierten Ergebnisse sind Integrations- und Inklusionsämter, Gewerkschaften und weitere Schulungsträger dazu aufgerufen, in ihren 
Weiterbildungsangeboten in stärkerem Maße als bisher Sozial- und Beratungskompetenz, die Rollenklärung unter Berücksichtigung eigener gesundheitlicher Beeinträchtigungen von Mitgliedern der SBV sowie die Zusammenarbeit von SBV und BR/PR zu thematisieren. Angesichts der aktuellen gesellschaftlichen Entwicklungen und der daraus resultierenden Dringlichkeit, die betriebliche Inklusion voranzubringen, sollten die Gewerkschaften darüber hinaus die SBVen in den Betrieben intensiver als bisher fördern und sie so in ihrer Rolle stärken. Die Gewerkschaften können die SBVen als wichtige Multiplikatoren in den Betrieben nutzen und auf betriebliche Entwicklungen Einfluss nehmen.

Insgesamt bleibt festzuhalten, dass die Schaffung einer inklusiven Arbeitswelt proaktiv gestaltet werden muss und die SBV dabei eine wichtige Funktion erfüllt. Unsere Ergebnisse unterstreichen die Bedeutung der Aufgabe, einen besonders schutzwürdigen Teil der Belegschaft zu vertreten und zu unterstützen, und sie belegen, dass die SBVen diese Aufgabe durch eine enge Zusammenarbeit mit ihrer Zielgruppe wahrnehmen. Gleichzeitig machen die SBVen durch die Repräsentanz der schwerbehinderten und ihnen gleichgestellten Beschäftigten die Relevanz von Chancengleichheit im betrieblichen Alltag besser sichtbar und leisten einen wichtigen Beitrag zur Sensibilisierung aller betrieblichen Akteur*innen. Die Stellung der SBV als Interessenvertretung der schwerbehinderten Beschäftigten sollte vor diesem Hintergrund weiter gefördert werden. In rechtlicher Hinsicht ist dies durch Änderungen im Bundesteilhabegesetz zwar ansatzweise geschehen, allerdings blieb die Reform hinter den Möglichkeiten zurück, sodass die Stärkung der Rechtsstellung der SBV ein Thema bleibt (Paul 2018). Schließlich richten unsere Ergebnisse die Aufmerksamkeit auch darauf, dass durch die Institutionalisierung des Amtes Spannungsverhältnisse in Form von Rollenkonflikten auftreten. Zur Optimierung der praktischen Tätigkeit und zur Stärkung der Stellung der SBV sind Angebote erforderlich, die diese Spannungen bearbeiten und aufzuheben versuchen.

\section{LITERATUR}

Bernhard, D. (2008): Weiterbildung betrieblicher Akteure im Kontext der beruflichen Integration behinderter Menschen. Eine Bildungsbedarfsanalyse, Aachen Bernhard, D./ Niehaus, M./ Marfels, B. (2011): Changes in managing disability in the workplace in Germany. Chances of professionalization?, in: Geisen, T./ Harder, H. G. (Hrsg.): Disability management and workplace integration. International research findings, Farnham, S. 57-70

BIH (Bundesarbeitsgemeinschaft der Integrationsämter und Hauptfürsorgestellen) (2018a): 10 Thesen des BIH - Vorschläge zur Weiterentwicklung des Rechts der Menschen mit Behinderung im beruflichen Kontext, Köln BIH (2018b): Die Schwerbehindertenvertretung, Wiesbaden

BMAS (Bundesministerium für Arbeit und Soziales) (2011): Übereinkommen der Vereinten Nationen über die Rechte von Menschen mit Behinderung - Convention of the United Nations on the rights of persons with disabilities, Bonn

Degener, T. (2009): Die UN-Behindertenrechtskonvention als Inklusionsmotor,

in: Recht der Jugend und des Bildungswesens 57 (2), S. 200-219

DGB (Deutscher Gewerkschaftsbund) (2015): Kölner Erklärung. Die

Schwerbehindertenvertretung als Motor der Inklusion, https://www.dgb. de/themen/++co++fgo6o79a-3b5a-11e5-91e6-52540023ef1a (letzter Zugriff: 12. 06. 2019)

Düwell, F. J. (2016): Welche Regelungen sind zur Sicherung der Rechte der Schwerbehindertenvertretungen geboten? Eine rechtspolitische Kontroverse, in Faber, U. / Feldhoff, K. / Nebe, K. / Schmidt, K. / Waßer, U. (Hrsg.): Gesellschaftliche Bewegungen - Recht unter Beobachtung und in Aktion, Festschrift für Wolfhard Kohte, Baden-Baden, S. 47-72

Düwell, F. J. (2018): Betriebsrat und Schwerbehindertenvertretung, in: Deinert, O. / Heuschmid, J./ Kittner, M. /Schmidt, M. (Hrsg.): Demokratisierung der Wirt- schaft durch Arbeitsrecht. Festschrift für Thomas Klebe zum 70. Geburtstag, Frankfurt a. M., S. 105-111

Flick, U./ von Kardorff, E. / Steinke, I. (Hrsg.) (2017): Qualitative Forschung. Ein Handbuch, Reinbek

INQUA (Initiative Neue Qualität der Arbeit) (2011): Altersdifferenzierte und altersgerechte Betriebs- und Tarifpolitik. Eine Bestandsaufnahme betriebspolitischer und tarifvertraglicher Maßnahmen zur Sicherung der Beschäftigungsfähig keit, Dortmund

ILO (Internationale Arbeitsorganisation) (2007): Gleichheit bei der Arbeit: Den Herausforderungen begegnen. Gesamtbericht im Rahmen der Folgemaßnahmen zur Erklärung der IAO über grundlegende Prinzipien und Rechte bei der Arbeit. Internationale Arbeitskonferenz, 95. Tagung 2007, Genf

Kahn, R. L. (1978): Konflikt, Ambiguität und Überforderung. Drei Elemente des Stress am Arbeitsplatz, in: Frese, M./ Greif, S. / Semmer, N. (Hrsg.): Industrielle Psychopathologie, Bern/Stuttgart/Wien, S. 18-33

Kahn, R. L. / Byosiere, P. (1992): Stress in organizations, in: Dunnette, M. D./ Hough, L. M. (Hrsg.): Handbook of industrial and organizational psychology, Palo Alto, S. 571-850

Kauffeld, S. (Hrsg.) (2014): Arbeits-, Organisations- und Personalpsychologie für Bachelor, Berlin

Kohte, W./ Liebsch, M. (2019): Neuregelungen im Schwerbehindertenrecht Stärkung der Schwerbehindertenvertretungen als Organ der Betriebsverfassung, in: Arbeit und Recht 1/2019, S. 4-10

Merton, R. K. (1973): Der Rollen-Set: Probleme der soziologischen Theorie., in: Hartmann, H. (Hrsg.): Moderne amerikanische Soziologie. Neuere Beiträge zur soziologischen Theorie, Stuttgart, S. 317-333

Nerdinger, F. W. (2012): Grundlagen des Verhaltens in Organisationen, Stuttgart Niehaus, M. / Heide, M. / Glatz, A. (2019): Von Anfang an zusammen. Handlungsleitfaden für Schwerbehindertenvertretungen in Netzwerken, Düsseldorf Paul, C. (2018): Die Schwerbehindertenvertretung als ein Organ der Betriebsverfassung, Berlin

Schiersmann, C./ Thiel, H.-U. (2018): Organisationsentwicklung. Prinzipien und Strategien von Veränderungsprozessen, Wiesbaden

Schumann, M. / Kuhlmann, M./ Sperling, H. J. (2008): Zwischen Toyota und Tradition. Das VW Projekt "Auto 5000" als mitbestimmungsjustierte Unternehmenskultur, in: Benthin, R. / Brinkmann, U. (Hrsg.): Unternehmenskultur und Mitbestimmung. Betriebliche Integration zwischen Konsens und Konflikt, Frankfurt a. M./New York, S. 243-258

Schuster, D. (2014): Die Schwerbehindertenvertretung als betrieblicher Interessenvertreter für Menschen mit Beeinträchtigungen - Aufgaben, Rechte und Pflichten. Diskussionsbeitrag Nr.14/2014, https://www.reha-recht.de/fachbeitrae ge/beitrag/artikel/diskussionsbeitrag-b14-2014 (letzter Zugriff: 12. 06. 2019)

Splanemann, A. (2002): Schwerbehindertenvertretung und Betriebsrat: Wege zur Kooperation, in: AiB - Arbeitsrecht im Betrieb 7/2002, S. 404-407

Stracke, S./ Nerdinger, F. W. (Hrsg.) (2009): „Alles unter einen Hut bringen?”. Rollen und Rollenkonflikte von Betriebsräten bei betrieblicher Innovation, Rostock

\section{AUTORINNEN}

MARIE SOPHIA HEIDE, M. A., wissenschaftliche Mitarbeiterin am Lehrstuhl für Arbeit und Berufliche Rehabilitation an der Universität zu Köln. Forschungsschwerpunkte: Gesundheit, Behinderung und inklusive Arbeitswelt, Rolle der Schwerbehindertenvertretung.

marie.heide@uni-koeln.de

MATHILDE NIEHAUS, Prof. Dr., Professur für Arbeit und Berufliche Rehabilitation an der Universität zu Köln. Forschungsschwerpunkte: Gesundheit, Behinderung und inklusive Arbeitswelt, Partizipationsforschung.

mathilde.niehaus@uni-koeln.de 